\title{
Analysis of angular distribution of fragments in relativistic heavy-ion collisions by quantum molecular dynamics
}

\author{
Tatsuhiko Ogawa, Tatsuhiko Sato, Shintaro Hashimoto, Koji \\ NiITA
}

Japan Atomic Energy Agency, Japan, Research Organization for Information Science and Technology, Japan

\begin{abstract}
To predict angular distribution of fragments produced in nucleusnucleus collisions, JAERI quantum molecular dynamics model (JQMD) was improved. Because JQMD underestimated fragments in the forward angle, which were mainly produced by peripheral collisions, JQMD was revised so as to simulate peripheral collisions accurately. Density-dependent in-medium effect and relativistic effect on nucleonnucleon interactions were incorporated for this purpose. The revised version of JQMD coupled with a statistical decay model was used to calculate differential fragment production cross sections measured in earlier studies. Comparison of the measured data and calculation by the revised and old JQMD showed that the revised JQMD can predict fragment angular distribution better than old JQMD. Particularly, agreement of fragment yield in the forward angle is substantially improved.
\end{abstract}

\section{Introduction}

The angular distribution of the fragments produced in nucleus-nucleus collisions is essential for cosmic ray physics, radiation safety of space exploration, and dosimetry in heavy-ion cancer therapy. 
Among the microscopic reaction models developed to simulate fragment production in nucleus-nucleus reactions, quantum molecular dynamics (QMD) models are generally adopted in general-purpose radiation transport simulation codes $[1,2]$ and used in various applications. One of the QMD models, JAERI-QMD (JQMD) [3] was developed as an event generator for general purpose radiation transport code PHITS (Particle and Heavy Ion Transport code System) [4] and widely used to simulate nucleus-nucleus collision (e.g., $[5,6])$. However, it was suggested that QMD models overestimated the width of the fragment angular distributions $[7,8]$ and underestimated production at the forward angle. This trend was probably attributed to inaccurate treatment of peripheral collisions, in which projectiles are little deflected and therefore fragments are ejected in the forward angle.

In this study, we revise JQMD to simulate the fragment angular distribution by accurate treatment of peripheral collisions.

Fragment production in the forward angle was underestimated by the previous version of JQMD; however, revised JQMD can reproduce fragment yield angular distribution measured in earlier studies.

\section{Methods}

In nucleus-nucleus collisions, the trajectory of the projectile ion is curved owing to the Coulomb repulsion by the target nucleus. The larger the impact parameter, the less is the fragment deflection.

In the previous version of JQMD; however, nuclei were sometimes spuriously disintegrated even without interacting with other nuclei owing to the instability of the nuclei simulated by JQMD. Therefore, nucleus-nucleus collisions at a large impact parameter simulated by JQMD may result in spurious reactions. To cut-off spurious reactions, impact parameter sampling range was intentionally truncated at a threshold defined by Eq. 1,

$$
b_{\max }=1.15 \times\left(A_{\mathrm{t}}^{1 / 3}+A_{\mathrm{p}}^{1 / 3}\right)-0.4(\mathrm{fm}),
$$

where $A_{\mathrm{t}}$ is target mass number and $A_{\mathrm{p}}$ is projectile mass number. This truncation cuts off not only spurious disintegration but also true peripheral collisions. As a result, fragments in the forward direction was underestimated.

The spurious decay of nuclei was occurred because the description of interaction between nucleons was frame-dependent. Nucleus configured to be stable in the rest frame became unstable in the center-of-mass frame of projectile and target nucleus. Therefore, Lorentz-covariant description 
of nucleon-nucleon interaction proposed by Mancusi [9] was adopted in the revised version of JQMD (hereafter referred to as JQMD-2.0). The equation of motion is described as follows;

$$
\begin{array}{r}
\dot{\mathbf{r}}_{\mathbf{i}}=\frac{\mathbf{p}_{\mathbf{i}}}{2 p_{i}^{0}}+\sum_{j}^{N} \frac{m}{p_{j}^{0}} \frac{\partial\left\langle\hat{V}_{j}\right\rangle}{\partial \mathbf{p}_{\mathbf{i}}}, \\
\dot{\mathbf{p}}_{\mathbf{i}}=-\sum_{j}^{N} \frac{m}{p_{j}^{0}} \frac{\partial\left\langle\hat{V}_{j}\right\rangle}{\partial \mathbf{r}_{\mathbf{i}}} . \\
p_{i}^{0}=\sqrt{\mathbf{p}_{\mathbf{i}}{ }^{2}+m^{2}+2 m\left\langle\hat{V}_{i}\right\rangle},
\end{array}
$$

where $\mathbf{r}_{\mathbf{i}}$ is the spatial coordinate of the centroid of the $i$-th nucleon, $\mathbf{p}_{\mathbf{i}}$ is the momentum of the $i$-th nucleon, $m$ is the rest mass of nucleons, $\left\langle\hat{V}_{j}\right\rangle$ is the potential of $j$-th particle, and $N$ is the number of particles in the system. In both JQMD and JQMD-2.0, the potential term of the Hamiltonian $V$ is a sum of the Skyrme-type force term, Coulomb interaction term, and symmetry term. The potential $V_{i}$ was inherited from previous JQMD without any modifications. Using the new equation of motion, nuclei at the ground state stayed stable over a typical reaction period of $150 \mathrm{fm} / \mathrm{c}$.

The impact parameter was sampled from zero to a threshold calculated by Eq. 1 in JQMD; however, impact parameter cut-off was not adopted in JQMD-2.0. Projectile and target stayed without losing nucleons or being substantially excited if the impact parameter was large enough. In this case, reaction simulation was started afresh after newly sampling the impact parameter.

In addition to the above improvements, density dependence of in-medium effect on nucleon-nucleon scattering cross sections was incorporated. Previously, we used neutron-proton scattering suppressed by in-medium Pauliblocking In JQMD; however, in-medium effect should get weaker near the nuclear surface, where nucleon-nucleon collisions are occurred in peripheral collisions. Therefore in JQMD-2.0, the neutron-proton scattering cross section in free space was adopted in peripheral collisions.

In actual simulation, JQMD and JQMD-2.0 were coupled with the statistical decay model GEM [10] to determine the final products. More details on JQMD-2.0 is provided elsewhere [11]. 


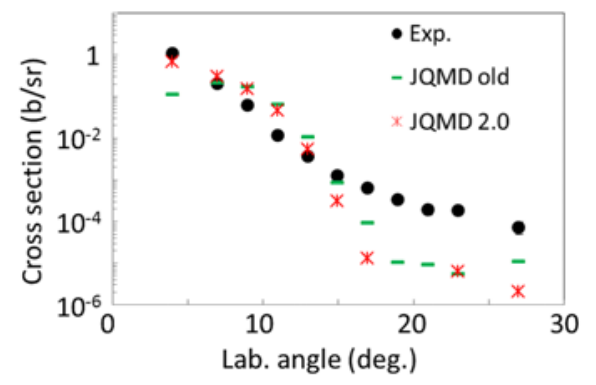

(a) ${ }^{12} \mathrm{C}\left({ }^{N a t} \mathrm{C}, \mathrm{x}\right){ }^{10} \mathrm{~B}$

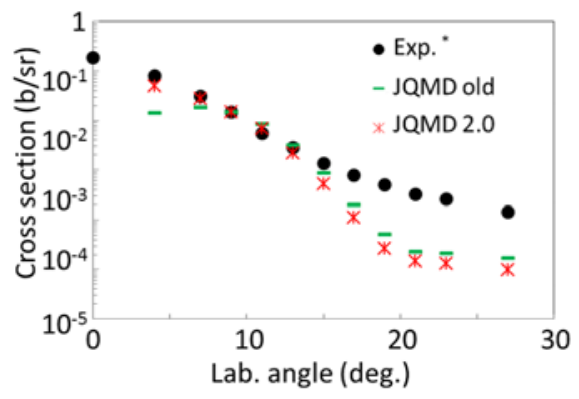

(b) ${ }^{12} \mathrm{C}\left({ }^{N a t} \mathrm{C}, \mathrm{x}\right){ }^{7} \mathrm{Be}$

Figure 1: Differential fragment production cross section of ${ }^{12} \mathrm{C}\left({ }^{N a t} \mathrm{C}, \mathrm{x}\right) \mathrm{X}$ at 95 $\mathrm{MeV} /$ nucleon calculated by JQMD, JQMD-2.0 and that measured by Dudouet et al. $[12,13]$.

\section{Results and discussion}

To evaluate the effect of the revisions, fragment production cross sections calculated by JQMD-2.0 were compared with measured data. Fragment production cross sections in ${ }^{12} \mathrm{C}\left({ }^{N a t} \mathrm{C}, \mathrm{x}\right)$ reactions, which are important for cosmic ray studies and cancer therapy, were used for this benchmark. Fig. 1 shows comparison of isotopic differential fragment production cross sections at $95 \mathrm{MeV} /$ nucleon calculated using JQMD, JQMD-2.0 and those measured by Dudouet et al. $[12,13]$.

The fragment yields calculated by JQMD have a peak at about 7 degrees and the yields are smaller at 4 degrees because peripheral collisions are disregarded by the impact parameter cut-off. In contrast, the fragment yields calculated by JQMD-2.0 are in better agreement with the measured data in this angular range. Particularly, ${ }^{10} \mathrm{~B}$ is produced by peripheral collisions in which two nucleons are knocked out, therefore the increase in the yield in the forward angle is more pronounced.

Above 15 degrees, yield is underestimated by both JQMD and JQMD2.0. Because the deflection of projectiles beyond 15 degrees cannot be explained by the dynamic reaction phase simulated by JQMD or the Coulomb repulsion, it is probably attributed to the statistical decay phase.

The increase in the yields at the forward angle and deficiency above 15 degrees were observed for the other isotopes.

Comparison of the differential fragmentation cross sections at a higher energy is shown in Figs. 2. Isotopic fragmentation cross sections of 


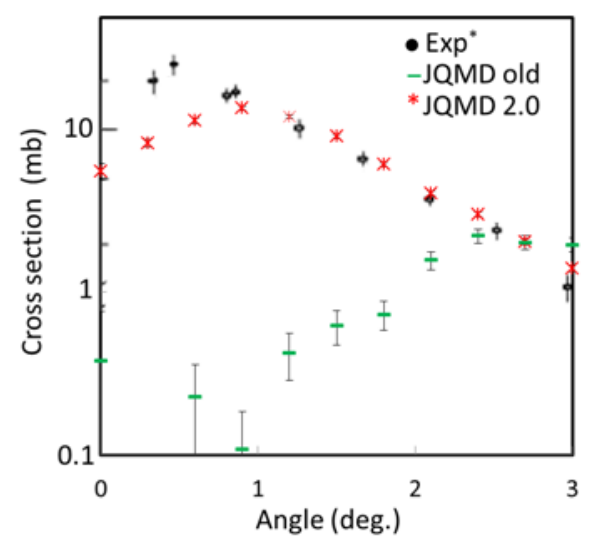

(a) ${ }^{12} \mathrm{C}\left({ }^{N a t} \mathrm{C}, \mathrm{x}\right){ }^{11} \mathrm{~B}$

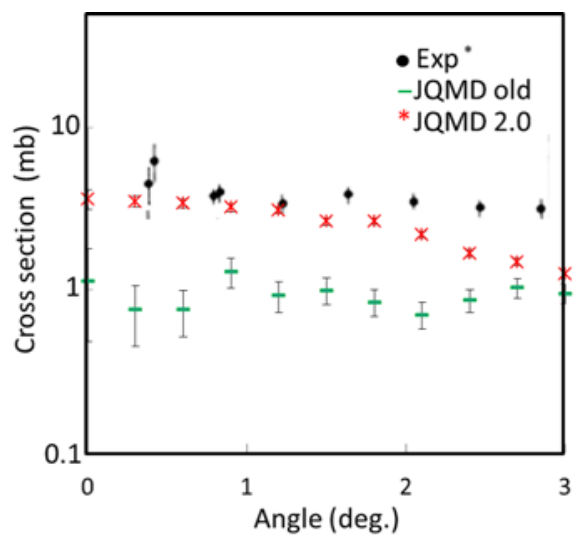

(b) ${ }^{12} \mathrm{C}\left({ }^{N a t} \mathrm{C}, \mathrm{x}\right){ }^{6} \mathrm{Li}$

Figure 2: Differential fragment production cross section of ${ }^{12} \mathrm{C}\left({ }^{N a t} \mathrm{C}, \mathrm{x}\right) \mathrm{X}$ at 250 $\mathrm{MeV} /$ nucleon calculated by JQMD, JQMD-2.0 and that measured by Kidd et al. [14].

${ }^{12} \mathrm{C}\left({ }^{N a t} \mathrm{C}, \mathrm{x}\right)$ reactions measured at $250 \mathrm{MeV} /$ nucleon [14] were compared with those calculated using the old JQMD and JQMD-2.0.

At higher energies, fragment angular distribution is more forward peaked owing to the decrease in the impulse of Coulomb repulsion.

In Fig. 2 (a), JQMD-2.0 reproduce the angular dependence of the yield below 2 degrees whereas the yield in the forward angle is underestimated by JQMD as with the case of Fig. 1.

On the other hand, the yield of ${ }^{6} \mathrm{Li}$ in Fig. 2 (b) is more angular independent compared to that of ${ }^{11} \mathrm{~B}$. This trend is reproduced both by JQMD and JQMD-2.0; however, JQMD underestimates the absolute yield by factor of 4 .

Above benchmark shows that JQMD-2.0 is more accurate than JQMD in reproducing the fragment yields at the forward direction.

\section{Conclusion}

Reaction event generator JQMD was revised for accurate simulation of fragment angular distribution in nucleus-nucleus collisions.

JQMD underestimated fragment yields at the forward angle owing to inaccurate treatment of peripheral collisions.

By introducing the Lorentz-covariant formulation of interaction, the ground state of target and projectile was stabilized. In addition, the density 
dependence of nuclear medium effect on proton-neutron scattering cross section was taken into account and the limitation of impact parameter sampling range was eliminated.

Comparison of the simulated results with earlier measurements showed that JQMD-2.0 reproduce fragment production in the forward angle more accurately. On the other hand, improvement of the statistical decay model is necessary for accurate simulation of fragment yield at large angles.

JQMD-2.0 has been incorporated to the general purpose radiation transport simulation code PHITS Ver. 2.76 and later. It can serve for a variety of applications such as cosmic ray physics and heavy-ion dosimetry.

\section{Acknowledgments}

We wish to thank Dr. Davide Mancusi for providing the source code of R-JQMD. We also thank the operation team of the Center for Computational Science and E-systems (CCSE) in the Japan Atomic Energy Agency. Monte-Carlo simulations reported in this paper were executed on a PCcluster system of CCSE. This work was partly supported by JSPS KAKENHI Grant Numbers 24860072 (Grant-in-Aid for Research Activity Startup), and 26790072 (Grant-in-Aid for Young Scientists B).

\section{References}

[1] F. Ballarini, G. Battistoni, F. Cerutti, A. Empl, A. Fasso, A. Ferrari, E. Gadioli, M.V. Garzelli, A. Ottolenghi, L.S. Pinsky, J. Ranft, S. Roesler, P. R. Sala, and G. Smirnov. Nuclear Models in FLUKA: Present Capabilities, Open Problems and Future Improvements, volume SLAC-PUB.

[2] T. Koi. New native QMD code in Geant4. Proceedings of Joint International Conference on Supercomputing in Nuclear Applications and Monte Carlo 2010 (SNA + MC2010).

[3] Koji Niita, Satoshi Chiba, Toshiki Maruyama, Tomoyuki Maruyama, Hiroshi Takada, Tokio Fukahori, Yasuaki Nakahara, and Akira Iwamoto. Analysis of the $\left(N, x N^{\prime}\right)$ reactions by quantum molecular dynamics plus statistical decay model. Phys. Rev. C, 52:2620-2635, Nov 1995.

[4] Tatsuhiko Sato, Koji Niita, Norihiro Matsuda, Shintaro Hashimoto, Yosuke Iwamoto, Shusaku Noda, Tatsuhiko Ogawa, Hiroshi Iwase, Hi- 
roshi Nakashima, Tokio Fukahori, Keisuke Okumura, Tetsuya Kai, Satoshi Chiba, Takuya Furuta, and Lembit Sihver. Particle and Heavy Ion Transport code System, PHITS, version 2.52. Journal of Nuclear Science and Technology, 50(9):913-923, 2013.

[5] L. Sihver, D. Mancusi, K. Niita, T. Sato, L. Townsend, C. Farmer, L. Pinsky, A. Ferrari, F. Cerutti, and I. Gomes. Benchmarking of calculated projectile fragmentation cross-sections using the 3-D, MC codes PHITS, FLUKA, HETC-HEDS, MCNPX-HI, and NUCFRG2. Acta Astronautica, 63(7-10):865 - 877, 2008.

[6] H. Yashima, Y. Uwamino, H. Iwase, H. Sugita, T. Nakamura, S. Ito, and A. Fukumura. Cross sections for the production of residual nuclides by high-energy heavy ions. Nuclear Instruments and Methods in Physics Research Section B: Beam Interactions with Materials and Atoms, 226(3):243 - 263, 2004.

[7] B. Alpat, E. Pilicer, S. Blasko, D. Caraffini, F. Di Capua, V. Postolache, G. Saltanocchi, M. Menichelli, L. Desorgher, M. Durante, R. Pleskac, and C. La Tessa. Total and partial fragmentation cross-section of 500 $\mathrm{MeV} /$ nucleon carbon ions on different target materials. Nuclear Science, IEEE Transactions on, 60(6):4673-4682, Dec 2013.

[8] J. Dudouet, D. Cussol, D. Durand, and M. Labalme. Benchmarking Geant4 nuclear models for hadron therapy with $95 \mathrm{MeV} /$ nucleon carbon ions. Phys. Rev. C, 89:054616, May 2014.

[9] Davide Mancusi, Koji Niita, Tomoyuki Maruyama, and Lembit Sihver. Stability of nuclei in peripheral collisions in the JAERI quantum molecular dynamics model. Phys. Rev. C, 79:014614, Jan 2009.

[10] S. Furihata. Statistical analysis of light fragment production from medium energy proton-induced reactions. Nuclear Instruments and Methods in Physics Research Section B: Beam Interactions with Materials and Atoms, 171(3):251 - 258, 2000.

[11] T. Ogawa, T. Sato, S. Hashimoto, D. Satoh, S. Tsuda, and K. Niita. Energy-dependent fragmentation cross sections of relativistic ${ }^{12} \mathrm{C}$. Phys. Rev. C, 92:024614, Aug 2015.

[12] J. Dudouet, D. Juliani, M. Labalme, D. Cussol, J. C. Angélique, B. Braunn, J. Colin, Ch. Finck, J. M. Fontbonne, H. Guérin, P. Henriquet, J. Krimmer, M. Rousseau, M. G. Saint-Laurent, and S. Salvador. 
Double-differential fragmentation cross-section measurements of $95 \mathrm{MeV}$ $\backslash$ nucleon ${ }^{12} \mathrm{C}$ beams on thin targets for hadron therapy. Phys. Rev. C, 88:024606, Aug 2013.

[13] J. Dudouet, M. Labalme, D. Cussol, C. Finck, R. Rescigno, M. Rousseau, S. Salvador, and M. Vanstalle. Zero-degree measurements of ${ }^{12} \mathrm{C}$ fragmentation at $95 \mathrm{MeV} \backslash$ nucleon on thin targets. Phys. Rev. C, 89:064615, Jun 2014.

[14] J. M. Kidd, P. J. Lindstrom, H. J. Crawford, and G. Woods. Fragmentation of carbon ions at $250 \mathrm{Mev/nucleon.} \mathrm{Phys.} \mathrm{Rev.} \mathrm{C,} \mathrm{37:2613-2623,}$ Jun 1988. 